\title{
Strong Coupling of a Spin Ensemble to a Superconducting Resonator
}

\author{
Y. Kubo, ${ }^{1}$ F. R. Ong, ${ }^{1}$ P. Bertet, ${ }^{1}$ D. Vion, ${ }^{1}$ V. Jacques, ${ }^{2}$ D. Zheng, ${ }^{2}$ A. Dréau, ${ }^{2}$ J.-F. Roch, ${ }^{2}$ A. Auffeves, ${ }^{3}$ F. Jelezko, ${ }^{4}$ \\ J. Wrachtrup, ${ }^{4}$ M. F. Barthe, ${ }^{5}$ P. Bergonzo, ${ }^{6}$ and D. Esteve ${ }^{1}$ \\ ${ }^{1}$ Quantronics group, SPEC (CNRS URA 2464), IRAMIS, DSM, CEA, 91191 Gif-sur-Yvette, France \\ ${ }^{2}$ LPQM (CNRS UMR 8537), ENS de Cachan, 94235 Cachan, France \\ ${ }^{3}$ Institut Néel-CNRS-UJF Grenoble, France \\ ${ }^{4}$ 3. Physikalisches Institut, Universität Stuttgart, 70550 Stuttgart, Germany \\ ${ }^{5}$ CNRS, UPR3079 CEMHTI, $1 D$ avenue de la Recherche Scientifique, 45071 Orléans, France \\ ${ }^{6}$ CEA, LIST, Diamond Sensors Laboratory, F-91191 Gif-sur-Yvette, France
}

(Received 28 June 2010; published 27 September 2010)

\begin{abstract}
We report the realization of a quantum circuit in which an ensemble of electronic spins is coupled to a frequency tunable superconducting resonator. The spins are nitrogen-vacancy centers in a diamond crystal. The achievement of strong coupling is manifested by the appearance of a vacuum Rabi splitting in the transmission spectrum of the resonator when its frequency is tuned through the nitrogen-vacancy center electron spin resonance.
\end{abstract}

DOI: 10.1103/PhysRevLett.105.140502

Building a quantum computer requires engineering a system that can reliably store quantum information and process it through a succession of quantum gates. Quantum bit implementations based on individual microscopic systems such as atoms, photons, electron or nuclear spins benefit from a natural decoupling from environmental noise, which results in long coherence times [1]; on the other hand, superconducting qubits [2] are macroscopic artificial atoms that couple strongly to electromagnetic fields, allowing faster single- and two-qubit gates [3], although with shorter coherence times. It is thus appealing to take the best of both worlds by combining artificial and natural quantum systems in "hybrid" quantum circuits that would exhibit long coherence times while allowing rapid quantum state manipulation. Whereas the coupling strength $g$ of an individual microscopic emitter to one electromagnetic mode is usually too weak to allow for coherent exchange of quantum information, the coupling strength of an ensemble of $N$ such systems is enhanced by $\sqrt{N}$ [4-6], allowing one to reach the strong coupling regime $g \sqrt{N} \gg \kappa, \gamma$, where $\kappa$ and $\gamma$ are the resonator and emitter damping rates. In that perspective, it has been proposed to couple an ensemble of cold molecules [7], atoms [8,9], or electron spins $[10,11]$ to a superconducting resonator that would mediate their interactions with one or a few superconducting qubits. This system would work like a genuine quantum Turing machine, with the ensemble providing a quantum memory [12-14] with long storage time, and the qubits providing the "hardware" to perform quantum gates [15].

Among all microscopic systems that can be coupled to superconducting circuits, negatively charged nitrogenvacancy centers $(\mathrm{N}-V)$ in diamond are particularly attractive [16]. They consist of a substitutional nitrogen atom and an adjacent vacancy having trapped an additional electron; their electronic ground state has a spin $S=1$
PACS numbers: 03.67.Lx, 42.50.Ct, 42.50.Pq, 85.25.Cp

with the states $m_{S}=0$ and $m_{S}= \pm 1$ separated by $\sim 2.87 \mathrm{GHz}$ in zero magnetic field [17]. The coherence time corresponding to this transition has been demonstrated to be as long as $2 \mathrm{~ms}$ at room temperature for samples isotopically enriched in ${ }^{12} \mathrm{C}$ [18]. Compared to atoms, $\mathrm{N}-V$ centers are perfectly compatible with superconducting circuits, because they do not require challenging trapping techniques or large magnetic fields to bring them in resonance at $\mathrm{GHz}$ frequency with the circuit. Finally, in addition to the electron spin resonance (ESR), $\mathrm{N}-V$ centers have two very interesting internal degrees of freedom: their narrow optical resonance might be used for coherently converting microwave into optical quantum states of the field [19], and their coupling to the nitrogen atom nuclear spin could give access to coherence times much longer than with electron spins [20].

In this Letter we report the observation of a vacuum Rabi splitting $[4,6,21,22]$ in the transmission spectrum of a superconducting resonator magnetically coupled to an ensemble of $\sim 10^{12} \mathrm{~N}-V$ centers with a collective coupling constant $g_{\text {ens }} / 2 \pi=11 \mathrm{MHz}$. This demonstrates the strong coupling of a microscopic spin ensemble to a macroscopic circuit. In a parallel experiment, Schuster et al. [23] observe a similar coupling to electronic spins in ruby and diamond (see also [24]). The sketch of our experiment is shown in Fig. 1. A diamond single crystal $(3 \times 3 \times$ $0.5 \mathrm{~mm}^{3}$ ) containing the $\mathrm{N}-V$ centers is glued on top of a half-wavelength niobium coplanar waveguide resonator, with a distance to the silicon substrate less than $\sim 0.5 \mu \mathrm{m}$ to ensure a maximum spin-resonator coupling. The diamond is positioned in the middle of the resonator where the magnetic field is maximum, with its (001) crystallographic plane facing the chip. The spin Zeeman splitting can be tuned with a magnetic field $\mathbf{B}_{\mathrm{N} V}$ parallel to the sample surface along the [100] axis within a few degrees. An array of four superconducting quantum inter- 

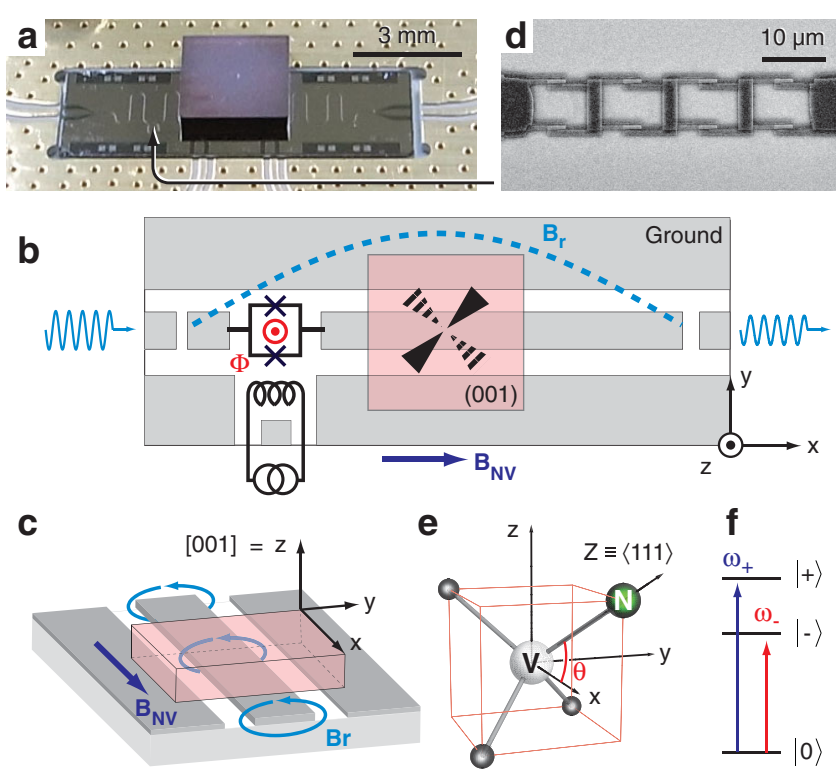

FIG. 1 (color). Scheme of the experiment. (a)-(c) Photograph, top and side view of the sample: a diamond crystal is glued on top and in the middle of a superconducting coplanar resonator with its (001) surface facing the chip. A SQUID array (d) is inserted in the central conductor to tune the resonator frequency $\omega_{r}(\Phi)$ with an on-chip wire producing a flux $\Phi$ in the SQUIDs. A magnetic field $\mathbf{B}_{\mathrm{N} V}$ is applied parallel to the [100] direction. (e) Sketch of a $\mathrm{N}-V$ center with its vacancy $(V)$ and nitrogen atom $(\mathrm{N})$, as well as three neighboring carbon atoms. Four equivalent $\langle 111\rangle$ directions exist for the $\mathrm{N}-V$ axis, all making the same angle $\theta=55^{\circ}$ with [100], and thus with $\mathbf{B}_{\mathrm{N} V}$. (f) Energy diagram of the $\mathrm{N}-V$ center, with the $\left|m_{S}=0\right\rangle$ ground state separated by $\omega_{ \pm}$from the excited states $| \pm\rangle$, which are linear combinations of the pure spin states $\left|m_{S}=+1\right\rangle$ and $\left|m_{S}=-1\right\rangle$.

ference devices (SQUID) is inserted in the resonator central conductor, away from the diamond crystal, to make its frequency $\omega_{r}(\Phi)$ tunable with the magnetic flux $\Phi$ threading the SQUID loops [25]. This flux is generated by passing current through an on-chip wire so that the resonator can be brought in resonance with the spins without changing their Zeeman splitting. The resonator transmission $\left|S_{21}\right|(\omega)$ is measured with a network analyzer, at powers low enough for the current through the SQUID to stay well below its critical current, so that the resonator behaves linearly [25].

We first characterize separately the coplanar resonator and the diamond crystal. A typical spectrum of the resonator alone, cooled at $40 \mathrm{mK}$, is shown in Fig. 2(a). The periodic dependence of the resonator frequency with $\Phi$ is shown in Fig. 2(b), in excellent agreement with the model described in [25] for parameters close to design values. On top of the modulation curve, the quality factor $Q=5 \times$ $10^{4}$ results from the combination of the coupling to the external line $\left(Q_{\text {ext }}=10^{5}\right)$ and from internal losses $\left(Q_{\text {int }}=\right.$ $10^{5}$ ). As already observed in similar samples, $Q$ decreases when the resonator is tuned to lower frequencies, possibly
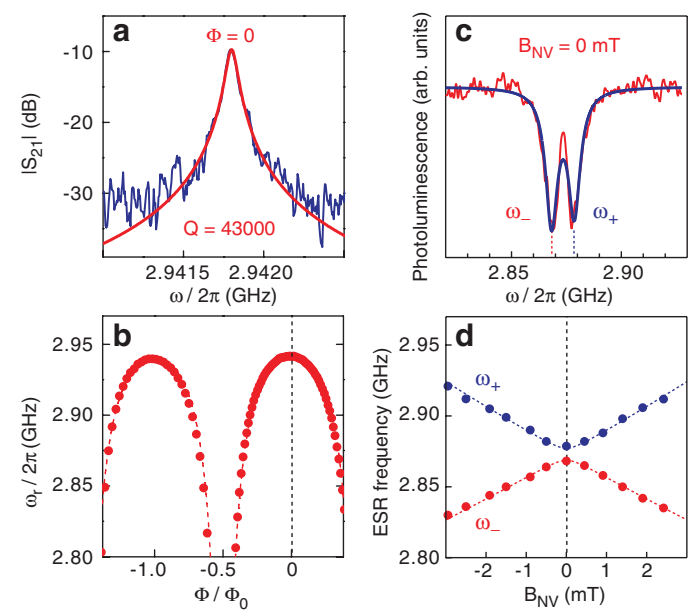

FIG. 2 (color). (a) Resonance line of the coplanar resonator with no diamond on top, measured at $40 \mathrm{mK}$. (b) Measured (dots) and fitted (dashed line) central frequency $\omega_{r}$ as a function of the applied flux $\Phi$, yielding a SQUID critical current $2 I_{C 0}=$ $2.2 \mu \mathrm{A}$ and a bare resonator frequency $\omega_{r 0} / 2 \pi=3.012 \mathrm{GHz}$. (c) Optically detected ESR of the $\mathrm{N}-V$ centers in the sample, in zero magnetic field and at room temperature. The $\omega_{ \pm}$lines are fitted by a sum of two $6 \mathrm{MHz}$ wide Lorentzians separated by 10.4 MHz. (d) Measured (dots) and fitted (dotted lines) dependence of $\omega_{ \pm}$on $B_{\mathrm{N} V}$, yielding a zero-field splitting $D / 2 \pi=$ $2.873 \mathrm{GHz}$ and a strain-induced splitting $E / 2 \pi=4.3 \mathrm{MHz}$.

due to flux noise or losses in the Josephson junctions [25]. Around the N-V center frequency of $2.87 \mathrm{GHz}$, we find $Q \sim 2 \times 10^{4}$ corresponding to a resonator energy damping rate $\kappa=\omega_{r} / Q \sim 0.9 \mathrm{MHz}$.

The diamond crystal (of the high pressure high temperature Ib type, with a nominal $100 \mathrm{ppm}$ nitrogen concentration) was irradiated with $2.5 \mathrm{MeV}$ protons at a dose of $5 \times 10^{16} \mathrm{~cm}^{-2}$ in order to create vacancies, and subsequently annealed at $900{ }^{\circ} \mathrm{C}$ for $10 \mathrm{~h}$ to form negatively charged $\mathrm{N}-V$ centers. The resulting $\mathrm{N}-V$ distribution is homogeneous over a $30 \mu \mathrm{m}$ depth from the irradiated surface and thus over the spatial extension of the resonator magnetic field (characteristic decay length of about $10 \mu \mathrm{m})$. The $\mathrm{N}-V$ concentration $\rho=(1.2 \pm 0.3) \times$ $10^{6} \mu \mathrm{m}^{-3}$ was measured by comparing the sample photoluminescence under a laser excitation at $532 \mathrm{~nm}$ to the photoluminescence of an individual center in the same conditions. A typical optically detected ESR spectrum is shown in Fig. 2(c): two resonance lines are observed even in zero magnetic field, due to residual strain in the crystal that lifts the degeneracy of states $\left|m_{S}=+1\right\rangle$ and $\left|m_{S}=-1\right\rangle$, yielding new eigenstates $|+\rangle$ and $|-\rangle$. The ESR frequencies measured as a function of $B_{\mathrm{N} V}$ [see Fig. 2(d)] are in quantitative agreement with the expected transitions $\omega_{ \pm}\left(B_{\mathrm{N} V}\right)$ between the energy eigenstates of the spin Hamiltonian $H_{\mathrm{N} V}=\hbar D S_{Z}^{2}+\hbar E\left(S_{X}^{2}-S_{Y}^{2}\right)+$ $g_{\mathrm{N} V} \mu_{B} \mathbf{S} \cdot \mathbf{B}_{\mathrm{N} V}$ [26], with a zero-field splitting $D / 2 \pi=$ $2.873 \mathrm{GHz}$, a strain-induced splitting $E / 2 \pi=4.3 \mathrm{MHz}$, a $\mathrm{N}-V$ Landé factor $g_{\mathrm{N} V}=2$, and $\mu_{B} / h=14 \mathrm{MHz} / \mathrm{mT}$. Here, $Z$ denotes the $\mathrm{N}-V$ axis [see Fig. 1(e)], while $X$ and 


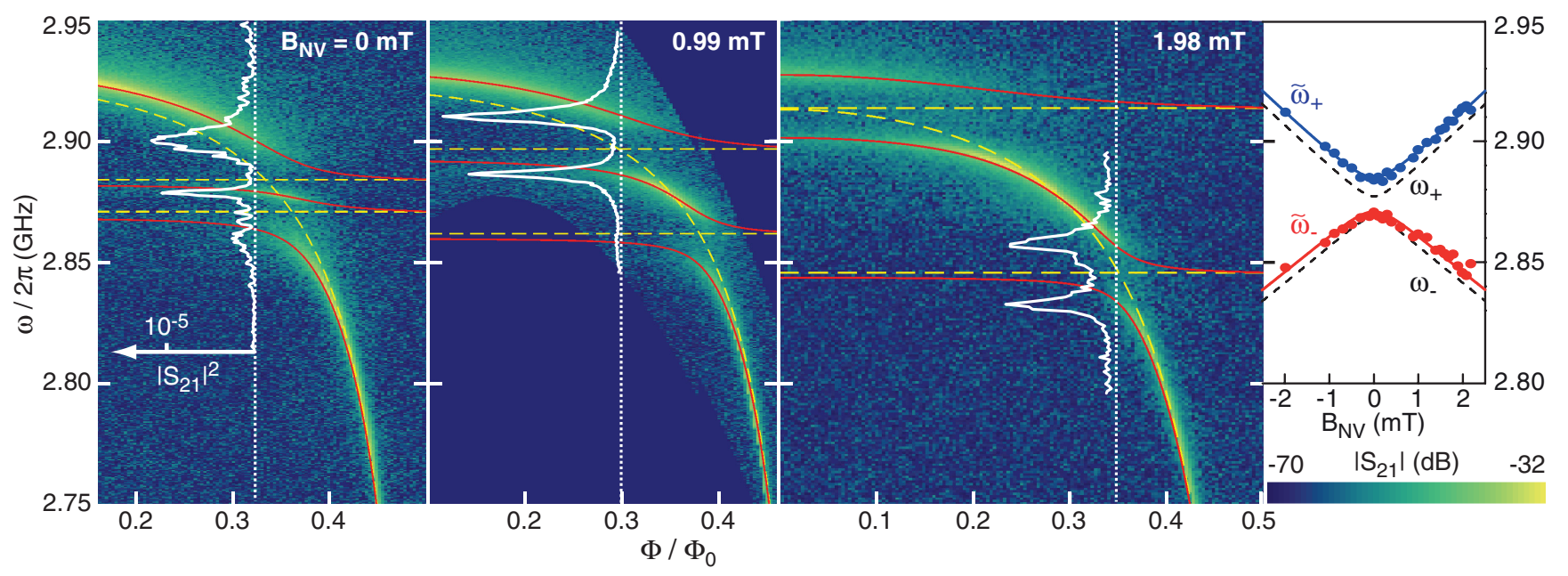

FIG. 3 (color). Transmission $\left|S_{21}\right|$ of the resonator with diamond on top (at $40 \mathrm{mK}$ ) as a function of $\Phi$ for $B_{\mathrm{N} V}=0$, 0.99, and $1.98 \mathrm{mT}$. Two anticrossings are observed symmetrically around $2.88 \mathrm{GHz}$, when the resonator frequency is resonant with the $\mathrm{N}-V$ transitions $|0\rangle \rightarrow|+\rangle$ and $|0\rangle \rightarrow|-\rangle$. Red solid (yellow dashed) lines are fits to the eigenfrequencies of the coupled (uncoupled) resonator-spin system as described in the text. A transmission spectrum (white overlay) is also shown in linear units in the middle of one of the two anticrossings, for each $B_{\mathrm{N} V}$ value. Right-hand panel: Experimental frequencies $\tilde{\omega}_{ \pm}$(dots) resulting from the fits of $\left|S_{21}\right|(\omega, \Phi)$ for several $B_{\mathrm{N} V}$ values. The data points are then fitted (solid lines) to the eigenfrequencies of Hamiltonian $H_{\mathrm{N} V}$ yielding $D / 2 \pi=2.878 \mathrm{GHz}$ and $E / 2 \pi=7.2 \mathrm{MHz}$. For comparison, the ESR frequencies $\omega_{ \pm}\left(B_{\mathrm{N} V}\right)$ measured at room temperature (see Fig. 2) are also shown as dashed lines.

$Y$ are the directions of nonaxial strain in the diamond matrix. Note that only two lines are observed in our geometry because the four possible $\mathrm{N}-V$ crystalline orientations are all at the same angle $\theta=55^{\circ}$ with the [100] direction and thus with $\mathbf{B}_{\mathrm{NV}}$. The measured ESR lines have a $\gamma / \pi \sim 6 \mathrm{MHz}$ FWHM linewidth, compatible with the expected broadening due to dipolar interactions with the neighboring nitrogen electronic spins $(S=1 / 2)$, given the nominal $100 \mathrm{ppm}$ nitrogen concentration [27].

We now present measurements of the resonator transmission with the diamond crystal on top. The microwave power used corresponds to a maximum intracavity energy of about 85 photons at resonance and is well below the value required to saturate the spins. Whereas optical pumping is required to observe the ESR at room temperature, cooling down the sample to $40 \mathrm{mK}$ already provides a strong spin polarization in $\left|m_{S}=0\right\rangle$. Two-dimensional plots of the transmission spectrum as a function of $\Phi$ are presented in Fig. 3 for several values of $B_{\mathrm{NV}}$, showing two avoided crossings when the resonator is tuned through the $\mathrm{N}-V$ center ESR frequencies. To account for these observations, we model the resonator-spin system [4] by the Hamiltonian $H / \hbar=\omega_{r}(\Phi) a^{\dagger} a+\tilde{\omega}_{+} b_{+}^{\dagger} b_{+}+\tilde{\omega}_{-} b_{-}^{\dagger} b_{-}+$ $\left(g_{+} a^{\dagger} b_{+}+g_{-} a^{\dagger} b_{-}+\right.$H.c), whose justification is given below. It describes the coupling of the resonator mode (annihilation and creation operators $a$ and $a^{\dagger}$ ) to two spin-wave modes (annihilation and creation operators $b_{ \pm}$ and $b_{ \pm}^{\dagger}$ ) representing the two ESR resonances at frequencies $\tilde{\omega}_{ \pm}\left(B_{\mathrm{N} V}\right)$, with coupling constants $g_{ \pm}$. The transmission spectrum $\left|S_{21}\right|(\omega)$ is fitted for each $\Phi$ by a sum of Lorentzian peaks, whose central frequencies are then fitted to the eigenfrequencies of $H$ (red solid lines in Fig. 3). At zero field, this fit yields $g_{+} / 2 \pi=g_{-} / 2 \pi=11.0 \pm$ $0.5 \mathrm{MHz}$ and a cooperativity parameter $C=g^{2} /(\kappa \gamma) \simeq$ 27 , which brings further confirmation that our experiment is in the strong coupling regime. The $\tilde{\omega}_{ \pm}\left(B_{\mathrm{N} V}\right)$ data points obtained and shown in Fig. 3 agree very well with the eigenfrequencies of the spin Hamiltonian $H_{\mathrm{N} V}$ for $E / 2 \pi=$ 7.2 $\mathrm{MHz}$ and $D / 2 \pi=2.878 \mathrm{GHz}$. This establishes that the anticrossings observed are indeed due to the magnetic coupling between the coplanar resonator and the $\mathrm{N}-V$ centers. The small changes in $E$ and $D$ with respect to the room-temperature values are likely due to thermal contraction upon cooling [28].

The coupled oscillator model also predicts that the widths of the two peaks at each anticrossing should be equal to the average of the resonator and spin linewidth. While this is approximately true at $B_{\mathrm{N} V}=1.98 \mathrm{mT}$, where the two lines have comparable linewidths of $\sim 7 \mathrm{MHz}$ in the middle of the anticrossing, this is clearly not the case at $B_{\mathrm{N} V}=0 \mathrm{mT}$, where the line in between the two anticrossings is much narrower than the other lines and narrower than the coupled model predicts (see Fig. 3). A more refined model is needed to understand this phenomenon. We also note that the $1.5 \mathrm{MHz}$ FWHM resonator linewidth (corresponding to $\kappa=9.4 \mathrm{MHz}$ ) in the presence of the diamond far away from the anticrossings is much larger than in Fig. 2(a), indicating that this particular diamond sample introduces losses of unknown origin.

We now justify our model for the interaction of the spin ensemble with the resonator. A single $\mathrm{N} V$ center with spin operator $\mathbf{S}_{k}$ located at position $\mathbf{r}_{k}$ is coupled to the resonator magnetic field $\mathbf{B}_{r}\left(\mathbf{r}_{k}\right)$ by the Hamiltonian $H_{k}=$ $g_{\mathrm{N} V} \mu_{B} \mathbf{B}_{r}\left(\mathbf{r}_{k}\right) \cdot \mathbf{S}_{k}$. Here $\mathbf{B}_{r}\left(\mathbf{r}_{k}\right)=\delta \mathbf{B}_{0}\left(\mathbf{r}_{k}\right)\left(a+a^{\dagger}\right)$ with 
$\delta \mathbf{B}_{0}\left(\mathbf{r}_{k}\right)$ the rms vacuum fluctuations of the magnetic field at $\mathbf{r}_{k}$. Restricting ourselves to one of the two $|0\rangle \rightarrow| \pm\rangle$ ESR transitions, this Hamiltonian can be put under a Jaynes-Cummings form $H_{k}=g_{k}\left(\mathbf{r}_{k}\right)\left(a \sigma_{+, k}+a^{\dagger} \sigma_{-, k}\right)$, with $g_{k}\left(\mathbf{r}_{k}\right)=\left(g_{\mathrm{N} V} \mu_{B} / \hbar\right) \delta \mathbf{B}_{0}\left(\mathbf{r}_{k}\right) \cdot\left\langle 0\left|\mathbf{S}_{k}\right| \pm\right\rangle$ and $\sigma_{+, k}$ $\left(\sigma_{-, k}\right)$ the raising (lowering) operator of the corresponding transition [29]. The coupling Hamiltonian of the ensemble of $N$ spins to the resonator is $H_{\text {ens }}=a \sum_{k} g_{k}\left(\mathbf{r}_{k}\right) \sigma_{+, k}+$ H.c, indicating that one excitation in the resonator mode is coupled to a well-defined coherent superposition of spin excitations (a spin wave) $[11,15]$. In the limit where the number of system excitations is small compared to $N$, this spin wave behaves as a harmonic oscillator described by an annihilation operator $b=\sum\left(g_{k}\left(\mathbf{r}_{k}\right) / g_{\text {ens }}\right) \sigma_{-, k}$, and the Hamiltonian can be rewritten as $H_{\text {ens }}=g_{\text {ens }}\left(a b^{\dagger}+a^{\dagger} b\right)$ with $g_{\text {ens }}=\left(\int \rho d \mathbf{r}|g(\mathbf{r})|^{2}\right)^{1 / 2}$. For a homogeneous distribution $\rho$ and a resonator and crystal of lengths $L$ and $l$, this collective coupling constant can be expressed as $g_{\text {ens }}=g_{\mathrm{NV}} \mu_{B} \sqrt{\eta \alpha \mu_{0} \hbar \omega_{r}(\Phi) \rho} / 2 \hbar$. Here, $\eta=(1 / L) \times$ $\int_{(L-l) / 2}^{(L+l) / 2} \sin ^{2} \frac{\pi x}{L} d x \quad$ and $\quad \alpha=\int\left|\delta \mathbf{B}_{0}(\mathbf{r})\right|^{2} \sin ^{2} \varphi(\mathbf{r}) d \mathbf{r} /$ $\int\left|\delta \mathbf{B}_{0}(\mathbf{r})\right|^{2} d \mathbf{r}$ are dimensionless factors describing, respectively, the fraction of the resonator mode volume occupied by the spins and their average orientation with respect to the resonator microwave field, $\varphi(\mathbf{r})$ being the angle between the $\mathrm{N}-V$ axis and $\mathbf{B}_{r}(\mathbf{r})$. We stress that apart from $\eta$ and $\alpha$, the ensemble coupling constant only depends on the spin density. For our sample, we estimate $\eta=$ 0.29 and $\alpha=0.81$, which combined with the measured $\mathrm{N}-V$ density yields $g_{\text {ens }} / 2 \pi=11.6 \mathrm{MHz}$, in agreement with the fitted value. This confirms that the spin ensemble is highly polarized and thus not far from thermal equilibrium.

Although the strong coupling regime was reached in this sample, the resonator and spin linewidths should be reduced by 1 order of magnitude in order to implement a quantum memory. This will require eliminating the extra microwave losses caused by the diamond crystal which are presently limiting the resonator linewidth. The spin resonance linewidth, on the other hand, is believed to be limited by dipolar interactions with neighboring nitrogen electronic spins; improving the nitrogen to $\mathrm{N}-V$ conversion rate would reduce the $\mathrm{N}-V$ linewidth while maintaining the large collective coupling constant measured here. The very same setup would then allow one to investigate the coherent oscillations between the spin ensemble and the resonator, using a rapid tuning of the resonator frequency [25] in and out of resonance with the spins. This would open the way to the storage and retrieval of a given microwave field at the single-photon level in the spin ensemble.

In conclusion, we have observed vacuum Rabi splittings in the transmission spectrum of a superconducting coplanar resonator magnetically coupled to an ensemble of $\mathrm{N}-V$ centers, with a collective coupling constant as large as $11 \mathrm{MHz}$. The position of these anticrossings is in excellent agreement with the electron spin resonances, and the measured coupling constant is well understood. These results therefore constitute an experimental evidence for the coherent coupling of a spin ensemble to a superconducting circuit, an essential step towards the implementation of more complex hybrid quantum circuits in which superconducting qubits, electron and nuclear spins, microwave and optical photons would be coherently coupled.

We gratefully thank M. Pomorski, N. Tranchant, G. Dantelle, F. Grosshans, N. D. Lai, F. Treussart, J. Botsoa, F. Lainé, F. Carrel, G. Balasubramanian, J. Meijer, S. Pezzagna, R. Kalish, D. Twitchen, D. Colson, and A. Forget for help in the diamond sample preparation, P. Sénat, J.-C. Tack, and P.-F. Orfila for technical help, and we acknowledge useful discussions with M. Afzelius, J. Isoya, and within the Quantronics group. We acknowledge the support of European Contract MIDAS.

[1] C. F. Roos et al., Phys. Rev. Lett. 92, 220402 (2004).

[2] J. Clarke and F. Wilhelm, Nature (London) 453, 1031 (2008).

[3] L. DiCarlo et al., Nature (London) 460, 240 (2009).

[4] M. G. Raizen et al., Phys. Rev. Lett. 63, 240 (1989).

[5] J. M. Fink et al., Phys. Rev. Lett. 103, 083601 (2009).

[6] Y. Kaluzny et al., Phys. Rev. Lett. 51, 1175 (1983).

[7] P. Rabl et al., Phys. Rev. Lett. 97, 033003 (2006).

[8] D. Petrosyan et al., Phys. Rev. A 79, 040304(R) (2009).

[9] J. Verdu et al., Phys. Rev. Lett. 103, 043603 (2009).

[10] A. Imamoglu, Phys. Rev. Lett. 102, 083602 (2009).

[11] J.H. Wesenberg et al., Phys. Rev. Lett. 103, 070502 (2009).

[12] M. Fleischhauer and M.D. Lukin, Phys. Rev. Lett. 84, 5094 (2000)

[13] C. Simon et al., Eur. Phys. J. D 58, 1 (2010).

[14] H. Wu et al., Phys. Rev. Lett. 105, 140503 (2010).

[15] K. Tordrup, A. Negretti, and K. Mølmer, Phys. Rev. Lett. 101, 040501 (2008).

[16] D. Marcos et al., arXiv:1001.4048; J. Twamley and S. D. Barrett, Phys. Rev. B 81, 241202 (2010).

[17] F. Jelezko et al., Phys. Rev. Lett. 92, 076401 (2004).

[18] G. Balasubramanian et al., Nature Mater. 8, 383 (2009).

[19] D. Englund et al., arXiv:1005.2204.

[20] M. V. Gurudev-Dutt et al., Science 316, 1312 (2007).

[21] Y. Zhu et al., Phys. Rev. Lett. 64, 2499 (1990).

[22] C. Weisbuch, M. Nishioka, A. Ishikawa, and Y. Arakawa, Phys. Rev. Lett. 69, 3314 (1992).

[23] D. Schuster et al., Phys. Rev. Lett. 105, 140501 (2010).

[24] I. Chiorescu et al., Phys. Rev. B 82, 024413 (2010).

[25] A. Palacios-Laloy et al., J. Low Temp. Phys. 151, 1034 (2008); M. Sandberg et al., Appl. Phys. Lett. 92, 203501 (2008).

[26] P. Neumann et al., New J. Phys. 11, 013017 (2009).

[27] J. A. van Wyk et al., J. Phys. D 30, 1790 (1997).

[28] V. M. Acosta et al., Phys. Rev. Lett. 104, 070801 (2010).

[29] The field in the middle of our resonator and $1 \mu \mathrm{m}$ above the surface is $\delta B_{0}=0.53 \mathrm{nT}$, yielding $g / 2 \pi=10.5 \mathrm{~Hz}$. 